Marialuisa Bottazzi

\title{
Kontinuität und Diskontinuität epigraphischer Praxis im Übergang von der Antike zum Mittelalter
}

In der antiken Welt kam der eingravierten, eingekratzten oder aufgemalten Schrift eine wichtige Rolle als Medium der Massenkommunikation zu. Mit ihr sollten mit möglichst breiter Wirkung und in dauerhafterem Material als Papyrus oder Pergament Ausdrücke unterschiedlicher menschlicher Erfahrung vermittelt werden, ganz gleich, ob diese nun individueller oder kollektiver, privater oder offizieller Natur waren.

Angesichts des Verlusts oder der zumindest äußerst fragmentarischen Überlieferung der öffentlichen Archive der antiken Städte stellen die Inschriften häufig die einzigen uns zur Verfügung stehenden Zeugnisse dar. Viele Inschriften liefern uns so Einsichten in die politische Organisation der Poleis. So haben etwa inschriftliche Zeugnisse die Existenz griechischer Kolonien in Afghanistan belegt, die in der handschriftlichen Überlieferung nicht erwähnt werden. Viele Inschriften bezeugen die politische Kultur der klassischen Welt, indem sie etwa wichtige Fälle von ProxenienVerleihung oder Euergetismus belegen, oder indem sie uns über die kulturellen und juristischen Traditionen von Orten informieren, die weit von den Zentren der römischen Verwaltung entfernt waren und über die ansonsten nichts bekannt wäre - man denke etwa an den Fall der großen, aus 17 Zeilen bestehenden griechischen Inschrift aus dem 2. Jahrhundert v. Chr., die von Michael Wörrle in Oinoanda, einer Stadt im antiken Lykien, ausgegraben wurde. ${ }^{1}$

\section{Von der antiken zur mittelalterlichen Epigraphik}

Schon die antiken griechischen und römischen Historiker zogen gerne epigraphische Zeugnisse heran, da diese als wichtige Primärquellen Informationen lieferten, die eine Rekonstruktion sowohl von Ereignissen als auch von biographischen Zusammenhängen ermöglichten. Dies gilt naturgemäß ebenso für das Verständnis der Eigenheiten der Gesellschaften, die diese Inschriften herstellten, und so den Faden einer kulturellen Tradition fortspannen und zugleich veränderten. Es ist in der Tat eine Art „Nabelschnur“ - so die treffende Formulierung von Lellia Cracco Ruggini ${ }^{2}$, die die epigraphische Kultur des klassischen Griechenlands mit der römischen, dann

1 Wörrle 1988.

2 Cracco Ruggini 1996.

Ә Open Access. (C) 2019 Marialuisa Bottazzi, publiziert von De Gruyter. (œ) Bł-Nc-ND Dieses Werk ist lizenziert unter der Creative Commons Attribution-NonCommercial-NoDerivatives 4.0 Lizenz.

https://doi.org/10.1515/9783110642261-005 
der spätantiken und schließlich der byzantinischen Epigraphik ${ }^{3}$ verbindet. Und ein ebenso wichtiger und klar zutage tretender Faden verbindet schließlich die antike Tradition mit der des Mittelalters, die durch den bekannten quantitativen Rückgang sowie die graphische Unsicherheit im Vergleich zum antiken Modell geprägt ist. Hierüber ist schon viel geschrieben worden. ${ }^{4}$

Die unzweifelhaften Metamorphosen, die bekannten Wiederverwendungen als Baumaterial sowie die natürlichen Verfallsprozesse haben jedoch die Spuren der epigraphischen Produktion zwischen dem 7. vorchristlichen Jahrhundert und der Spätantike nicht ausgelöscht, so dass man bis in das Frühmittelalter hinein eine Kontinuität epigraphischer Praxis beobachten kann; und so nimmt der (methodische) Weg, den die sich mit Inschriften beschäftigenden Historiker normalerweise nehmen, um für Italien die epigraphische Kontinuität zwischen der Spätantike und dem Mittelalter nachzuzeichnen, seinen Ausgang bei den großen epigraphischen Traditionen Roms, die durch eine vielfältige Typologie geprägt war, wie sie etwa Alfredo Buonopane umrissen hat. $^{5}$

Es ist unmöglich, sich der mittelalterlichen Epigraphik Italiens zu nähern, ohne die Arbeiten von Armando Petrucci zu berücksichtigen, der seit den 1970er-Jahren unter paläographischen und insbesondere historischen Gesichtspunkten die Spezifika der epigraphischen Tradition Italiens, sowohl im Allgemeinen als auch unter besonderer Berücksichtigung der Funeral-Epigraphik, bestimmt hat. ${ }^{6}$ In den letzten Jahren haben, ausgehend von den Arbeiten Petruccis, die hervorragenden Beiträge von Flavia De Rubeis die Eigenheiten der epigraphischen Kontinuität zwischen Spätantike und Frühmittelalter thematisiert, wodurch sie vor allem der Erforschung der langobardischen Epigraphik (vor allem in Süditalien) wichtige Impulse gab. Carlo Carletti hingegen hat eine der klarsten Gesamtinterpretationen der christlichen Epigraphik vom 3. bis 7. Jahrhundert vorgelegt. ${ }^{7}$

Die von den zitierten Autoren erarbeitete und hier in groben Zügen umrissene epigraphische Entwicklung nach dem Niedergang des Römischen Reichs war das Ergebnis der wohlbekannten rasanten politischen und kulturellen Entwicklung, wie sie von Paolo Cammarosano für den Westen und Cécile Morrisson für den Osten eindrücklich beschrieben wurde. ${ }^{8}$ Wirtschaftliche und soziale Krisen, Pandemien, Hungersnöte, Erdbeben, Invasionen und Kriege wirkten sich zusammen mit der „Chris-

3 Cavallo 1988, 467-516; Cavallo/Mango 1995, VII-XIV. Die byzantinische Epigraphik ist Gegenstand der eindrucksvollen Arbeit von Antonio Enrico Felle über die Verwendung biblischer Texte in öffentlichen Inschriften zwischen der Spätantike und der frühen byzantinischen Zeit (4. bis 8. Jahrhundert): Felle 2015, 353-370.

4 Siehe hierzu auch die Beiträge von Nicoletta Giovè und Katharina Bolle in diesem Band.

5 Buonopane 2009.

6 Petrucci 1995.

7 Carletti 2008, 7.

8 Für eine politische Übersicht des Westens siehe Cammarosano 1999; für den Osten Morrisson 2007, 5-52. 
tianisierung der Epigraphik“ auf die inschriftliche Praxis aus, so dass kurz nach der Mitte des 6. Jahrhunderts, in den wenigen Jahren zwischen Justinians Tod (565) und dem Beginn der Zersplitterung des mittelalterlichen Italiens nach der langobardischen Eroberung, die Verwendung von eingemeißelter Schrift bereits stark begrenzt war. So verschwanden Texttypen, die zuvor in römischer Tradition auch inschriftlich gefasst worden waren; zudem beruhte die gleichwohl erkennbare inschriftliche Kontinuität auf christlichen Auftraggebern und Produzenten.

Obwohl der Hauptbereich, in dem die Inschriftenverwendung gefördert wurde, seit geraumer Zeit der christliche war, bestand doch zugleich eine Inschriftenproduktion im Bereich weltlicher Institutionen fort. ${ }^{9}$ Doch der Überlebensprozess der epigraphischen Ausdrucksformen, aus denen sich schließlich seit dem 4. Jahrhundert die mittelalterliche Epigraphik entwickelte, beruhte bereits ausschließlich auf den kirchlichen und monastischen Eliten. Diese Eliten waren im Zuge der breiten und fortschreitenden Auflösung der sozialen, politischen und institutionalen Ordnung der Spätantike bereits durch die Entscheidung Konstantins, große Teile der kulturellen und territorialen Organisation der Diözesen an die Kirche zu übertragen, offiziell beauftragt worden. ${ }^{10}$ Hier müssten weitere Bemerkungen folgen, um die Kontakt- und Transformationspunkte zwischen der römisch-laikalen und römisch-christlichen Epigraphik während des 4. Jahrhunderts herauszuarbeiten. Da ich hier zunächst eine Übersicht geben möchte, verschiebe ich entsprechende Bemerkungen sowie eine allgemeine Charakterisierung der mittelalterlichen Entwicklung von eingravierter Schrift aber an das Ende meiner Ausführungen. Erwähnt werden soll hier nur, dass die Übertragung eines Großteils der administrativen Leitungsfunktionen an die Bischöfe in kurzer Zeit nicht allein die bereits geschwächte römische Sozialordnung, in der sich zunehmend neue Hegemonialgruppen bildeten, ${ }^{11}$ tiefgreifend prägte - sie bedingte auch auf dramatische und einschneidende Weise einen regelrechten Kurswechsel in der Auseinandersetzung mit der antiken Kultur.

Mitte des 6. Jahrhunderts, mithin am Beginn meines Untersuchungszeitraums, hatten schon alle bekannten Entwicklungen eingesetzt, die durch das Eindringen der christlichen Orthodoxie in das soziale und politische Leben in Gang gesetzt wurden. Dieses Eindringen ist als Ausdruck des Willens von Kaiser Justinian zu werten, der mehrere öffentliche Funktionen an Bischöfe übertragen hatte, die seitdem über richterliche Kompetenzen verfügten und überdies die Ausführung der erlassenen kaiserlichen Gesetze beaufsichtigten und kontrollierten. Zugleich war die Praxis verbindlich gemacht worden, dass dieselben die erlassenen Gesetzestexte zum Zwecke einer weiten Verbreitung veröffentlichen sollten.

9 Feissel 1995, 67-98; Lepellet 1997, 335-352; Roueché 1997, 353-368; Orlandi 2006, 311-324; Porena 2006, 325-356; de Santiago Fernàndez 2009, 291-344; Lepore 2012.

10 Für eine politische Übersicht des Westens siehe Cammarosano 1999, 3-48; Flussin 2007, $119-151$. 11 Cammarosano 1999, 19-25; Morrisson 2007, 28. 
Die Gesetzgebung, die Justinian seit $535 \mathrm{zu}$ promulgieren begann (Novellae Constitutiones), bezog sich zugleich auch auf den Bereich der Kommunikation, indem die öffentliche Bekanntmachung der Gesetze in der Form von Inschriften erfolgen musste, die an von kaiserlicher Seite bestimmten Orten veröffentlicht werden sollten. Mit der am 20. Mai 535 verabschiedeten Novella VIII, die später auch für das gesamte Gebiet des Imperiums bestätigt wurde (das Italien der Pragmatica Sanctio pro petitione Vigilii von 554 eingeschlossen), wurden die Innenräume der Kirchen und deren Portiken als der von der öffentlichen Gewalt angegebene Bereich festgelegt, in dem Verordnungen feierlich vermittelt werden sollten. ${ }^{12}$ Diese Orte hielt Justinian für am geeignetsten, um Holztafeln oder -platten aufzunehmen, auf denen die Gesetzestexte so wiedergegeben werden mussten, dass alle ihren Inhalt verstehen konnten, „cumque lex publica proposita fuerit et omnibus manifestata (...), utpote dicata deo et ad salutem ab eo factorum hominum scripta“.${ }^{13}$ Man könnte sagen, dass dieser Vorgang unauslöschlich das gesamte epigraphische Mittelalter geprägt hat.

Aufgrund des Zerfalls des römischen Systems, in das die Kirche eingedrungen war, erschien die Kultur Mitte des 6. Jahrhunderts folglich bereits sehr weit entfernt „vom liberal-aristokratischen Geist der antiken gebildeten Gesellschaft““. ${ }^{14}$ Gleichwohl war für die zum Mittelalter führende Entwicklung das Bildungsangebot der Bischofsschulen nicht von geringerer Bedeutung. ${ }^{15}$ Das gesamte System der Ausbildung und der gelehrten Textproduktion, in das natürlich auch die epigraphische Produktion eingeschlossen war, entwickelte sich zum ausschließlichen Erbe der religiösen Eliten, während sich die hervorgebrachten Texttypen unmittelbar auf deren Bedürfnisse beschränkten, begründeten diese ihre Macht doch auf der Memoria, auf Ritualen und Symbolen, natürlich immer in der jeweiligen Formung durch die christliche Ideologie.

Die unmittelbare laikale Verwendung der Epigraphik gehörte nun bereits einer vergangenen Tradition an. Erst die oft beschworene Renaissance des 12. Jahrhunderts, die alle Bereiche der Kultur betraf, bezeichnenderweise auch die der Rechtswissenschaft, war auch eine „epigraphische Renaissance“. So wurden die lateinischen Klassiker und die Texte der klassischen Rechtswissenschaft erneut zur Basis einer fundierten intellektuellen Bildung von Gelehrten und Politikern. In einem regelrechten Crescendo entstanden seit der Mitte des 12. Jahrhunderts zahlreiche inschriftliche Texte mit besonderer Relevanz für das kommunale Leben. Anfänglich wurden sie an den Außenwänden von Kathedralen angebracht. Als Beispiel sei auf die große Inschrift der Statuten von Ferrara von 1173 verwiesen. ${ }^{16}$ In dieselbe Phase ist auch

12 Für einen ausschließlich mittelalterlichen Überblick über die Verwendung von Inschriften und ihren Raum: Petrucci 1985, 85-97.

13 Cod. Iust., Nov. VIII, Edictum, Bd. 3.

14 Auerbach, 1958, 191.

15 Auerbach 2007, 227-231.

16 Franceschini 1969. 
eine Reihe feierlicher städtischer Bauinschriften einzuordnen, die nicht mehr allein den kirchlichen Räumen vorbehalten waren: etwa die Inschriften für den Ponte dei Molini in Modena von 1190, die sienesische Inschrift für die Porta Fontebranda von 1193 und jene spätere, noch heute sichtbare, die auf den Mauern von Monteriggioni ausgestellt und auf das Jahr 1214 datiert ist. ${ }^{17}$ Den absoluten Höhepunkt bildet in dieser Hinsicht das 14. Jahrhundert, für das der Anonimo Romano an den gebildeten Umgang Colas di Rienzo mit den „antiqui pataffi“ erinnert, mit denen letzterer dem römischen Volk zeigte, welches Verhältnis und welche Kontinuität zwischen den antiken und den neuen städtischen Institutionen bestand. ${ }^{18}$

\section{Brüche und Kontinuitäten}

Zwar kann man die römische Produktion als Höhepunkt einer vollständig säkularen Verwendung der Epigraphik auffassen, doch auch in hochmittelalterlicher Zeit war der Gebrauch der eingravierten Schrift durch Laien wieder weit verbreitet. Im Vergleich dazu lässt sich feststellen, dass die Verwendung von Inschriften durch Laien im Frühmittelalter zwar kontinuierlich - wenn auch quantitativ sehr begrenzt - belegt ist, jedoch ausschließlich in dem Gebiet, das zum byzantinischen Italien gehörte. Im nördlichen Italien hingegen sind die eingravierten Texte auf den ausschließlichen Gebrauch als Grabinschriften oder aber als feierliche Gedenkinschriften begrenzt, die im Zusammenhang mit der Gründung oder Stiftung von Kirchen oder Klöstern durch Angehörige der weltlichen Eliten des langobardischen, ${ }^{19}$ später des karolingischen Italien entstanden. ${ }^{20}$ Auch deren Entstehungsumstände lassen sich allerdings als kirchlich bezeichnen. Die sich anschließende karolingische Phase stellte diesbezüglich keine Veränderung dar, da sie im Bereich der Memoria durch die Kontinuität mit der langobardischen Zeit geprägt war. Im Gegenteil war diese umso mehr auf die Kultur der Bischöfe, Äbte und Mönche ausgerichtet, denen die Führungsgruppen ihre Epitaphe und damit ihre Seelen anvertrauten.

An dieser Stelle sind zwei Aspekte, die De Rubeis in ihren Arbeiten zur langobardischen und karolingischen Epigraphik vorschlägt, besonders hervorzuheben: Die im Italien des 7. und 8. Jahrhunderts für die Mitglieder der Eliten angefertigten Epitaphe bedienten sich in vielen Fällen einer erhabenen bzw. höfischen Schrift. Diese ist durch ein hohes graphisches und dekoratives Niveau charakterisiert und zudem von

17 Bottazzi 2006, 305-360; dies. 2007, 272-306; dies. 2012, 275-302; dies. 2015, 47-60; dies. 2016, 25-53; dies. 2017.

18 Porta 1979, 143; Cammarosano 2000, 301, 307, 311.

19 De Rubeis 2000, 139-173.

20 Für die langobardische Produktion allgemein: De Rubeis 2003, 481-506; dies. 2006, 99-126. Zur Inschriftenkultur der Langobarden siehe jetzt Rotili 2015, 75-144 (insbesondere zur Epigraphik S. 114119). 
einem textuellen Gehalt, der sie zu einem regelrechten Modell für die Repräsentation eines „Geblütsadels“ (nobiltà di stirpe) macht. Dies gilt sicher für die wundervollen Inschriften, die König Cunibert und seiner Tochter, der Äbtissin Cuniberte, gewidmet sind. Nur wenige Zeit früher zeichneten derartige Inschriften den Adligen Aldo und etwas später den Abt Cummiano von Bobbio aus. Doch die Tradition derartiger inschriftlicher Repräsentation der Eliten lässt sich nur in der Langobardia Minor nachweisen, während im Gebiet von Mailand, in Fermo für den Dux Tasbuno oder in Viterbo für Alperga, die Tochter des Königs Desiderius, querformatige InschriftenSteine oder -Platten verwendet wurden, auf denen der Text in einer groben Kapitalis von sehr unregelmäßigem Modulus eingemeißelt wurde, ohne irgendwelchen Vorzeichnungen oder Liniierungen $\mathrm{zu}$ folgen. ${ }^{21}$

Die zweite Überlegung von De Rubeis betrifft die karolingische InschriftenProduktion, bei der sie keine erkennbare Orientierung an irgendeinem graphischen Modell, hingegen eine kontinuierlich hohe Qualität der Textgestaltung beobachtet.

Hinzuzufügen ist meiner Ansicht nach eine dritte Beobachtung, die bei einer Thematisierung der frühmittelalterlichen epigraphischen Kontinuitäten einzubeziehen ist. Diese Beobachtung betrifft den Umgang mit Inschriften, der sich bei der zweiten und dritten Generation derjenigen Eliten nachweisen lässt, die sich in der Folge der Etablierung der Herrschaft Karls des Großen im Regnum in Italien niedergelassen und sich in den unterschiedlichen Gebieten verwurzelt haben, wo sie verschiedene Ämter und Titel erwarben. Gleich, ob es sich um Grafen oder allgemeiner um vassi, um milites oder inlustri viri, etwa die Erben des Grafen Leone di Como, von Ermenulfo und Eremberto oder später des Iohannes senior von Ceneda handelt, alle diese neuen Generationen gründeten, vergleichbar den höchsten Eliten des Reichs, aus eigenen Mitteln Kirchen und Klöster. Und es war diesen stets wichtig, dass dabei das eigene Werk, die Vorfahren oder die eigene Person in Erinnerung bewahrt würde. Dass dies mit epigraphischen Mitteln erfolgte, belegt somit, dass die Traditions-Inschrift als Medium der Kommunikation, der Sichtbarmachung und der sozialen Nobilitierung bereits durch viele, wenn nicht alle ,Familien` des Regnums übernommen worden war. ${ }^{22}$

Eine ganz andere Situation kennzeichnet den Süden Italiens, wo der Inschriftengebrauch bereits im 8 . Jahrhundert kontinuierlich und auf hohem qualitativem Niveau erfolgte. Dieser ähnelte in vielfacher Hinsicht demjenigen der langobardischen Phase in den norditalienischen Regionen, doch war er durch eine vielfältigere Typologie geprägt, insbesondere durch den bewussten Gebrauch von Urkundenin-

21 Carosi 1986; Cammarosano 2015; Rotili 2015, 114-119.

22 Bottazzi 2012, 89-108, 319-226. Untersuchungen zu den Familien des Regnum und später den kommunalen Familien habe ich im Rahmen einer prosopographischen Arbeit angestellt, die noch in Druck ist und von Ermanno Arslan herausgegeben wird. Sie ist der Porta Romana von Mailand gewidmet. 
schrifen (carte lapidarie): ${ }^{23}$ ein Inschriftentypus, der sich vollständig an die Notariatsinstrumente angleichen konnte, aus denen er hervorging. Urkundeninschriften wurden, soweit wir wissen, in Norditalien erst seit dem Anfang des 11. Jahrhunderts angefertigt, wo sie regelmäßig an sakralen Orten angebracht wurden. ${ }^{24} \mathrm{Im}$ Italien der Langobardia minor sind sie hingegen durch eine im Staatsarchiv von Neapel aufbewahrte Platte bereits seit dem 8. Jahrhundert gut bezeugt. Auf dieser Platte wurde, wahrscheinlich als Ergebnis byzantinischer Einflüsse, eine Zusammenfassung der vom Notar Giuliano verfassten charta venditionis eingemeißelt, die die Grenzen und Zugehörigkeiten des im Gebiet von Cuma gelegenen Hofes Memorola festlegte. ${ }^{25}$

Im Norden Italiens war somit für einen Großteil des Mittelalters bis weit ins 13. Jahrhundert hinein, als erstmals deutlichere und dauerhaftere Zeichen einer Renaissance der weltlichen Kultur in Erscheinung traten, die kulturellen Rahmenbedingungen einer Verwendung von Inschriften kirchlich geprägt. Bestimmt waren sie zwar auch durch Momente großer Gelehrsamkeit, diese blieben jedoch Episoden und waren noch zu exklusiv, um erkennbar eine Kontinuität des Inschriften-Gebrauchs zu belegen. Gleichwohl lassen diese Spuren des Gebrauchs und des kontinuierlichen Interesses an den Inschriften, dokumentiert durch die sporadischen Zeugnisse des 6., 7. und 8. Jahrhunderts sowie durch handschriftliche Sammlungen von Inschriftentexten, meiner Meinung nach erkennen, dass die Traditionslinien doch weniger isoliert waren als die Forschung bisher angenommen hat. Eine wichtige Hilfe auf diesem Weg stellen die Arbeiten von Claudia Villa dar, die uns in den letzten zwanzig Jahren mehrfach aufgezeigt hat, dass die lateinische Kultur des Frühmittelalters deutlich fundierter und verbreiteter war, als man gemeinhin annehmen möchte.

Als Indiz für diese besondere Aufmerksamkeit für die epigraphische Kultur können die zahlreichen Inschriftensammlungen gewertet werden, die beginnend mit dem 4. Jahrhundert im gesamten Frühmittelalter angelegt und mit dem Ziel konzipiert und organisiert wurden, als exempla der Instruktion von gebildeten Verfassern und Schreibern von Inschriften zu dienen. ${ }^{26}$ Für die Suche nach Kontinuitäten können wir neben diesen Sammlungen epigraphischer Texte auch auf zahlreiche weitere Handschriften verweisen, in denen ebenfalls einzelne Inschriftentexte transkribiert wurden, sei es aus literarischem Interesse oder im Zusammenhang mit annalistischen oder kalendarischen Aufzeichnungen. ${ }^{27}$ Derartige Transkriptionen findet man nicht selten in Sammelhandschriften in den großen Handschriftenbeständen, etwa in der

$23 \mathrm{Zu}$ dem Typus der Urkundeninschriften siehe ausführlich den Beitrag von Vincent Debias in diesem Band.

24 Bottazzi 2012a, 331-368; dies. 2012c, 501-522.

25 Capasso 1892, Bd. 2.2, 292-293. Für die langobardische Produktion in Süditalien verweise ich auf die Aufsätze von De Rubeis 2003; Ferraiulo 2013; Lambert 2008; ders. 2012, 99-114.

26 Sanazzaro 2006, 81-96. Siehe hierzu auch den Beitrag von Katharina Bolle in diesem Band.

27 Bottazzi 2016, 89-111 zu Fällen von Transkriptionen von Inschriften aus St. Gallen und San Michele della Chiusa, die wegen ihrer Memorial-Funktion von besonderer Bedeutung sind. 
Biblioteca Apostolica Vaticana oder der Stiftsbibliothek von St. Gallen. ${ }^{28}$ Ähnliche Motive wie bei den gebildeten Kompilatoren und Historiographen lassen sich auch bei Agnellus von Ravenna erkennen, dem Verfasser des im 9. Jahrhundert entstandenen Liber pontificalis ecclesiae Ravennatis. ${ }^{29}$ In diesem wurden die Biographien aller Erzbischöfe von Ravenna von der Mitte des 5. Jahrhunderts (Bischof Apollinaris) bis zur Mitte des 9. Jahrhunderts (Bischof Georg) zusammengestellt, wobei der Autor sich auf unterschiedliche Quellen bezog, unter denen mit besonderer Häufigkeit epigraphische Zeugnisse hervorragen. Insgesamt transkribierte er 50 epigraphische Texte und zitierte weitere 30, die durch Abnutzung, ungünstige Anbringungssituationen oder andere Gründe von ihm nicht gelesen werden konnten, deren Existenz er so jedoch bezeugt. Agnellus ist für uns eines der überzeugendsten frühmittelalterlichen Beispiele für die kontinuierliche Aufmerksamkeit, die man der epigraphischen Produktion schenkte, da dieser für seine historiographische Rekonstruktion wesentlich auf inschriftliches Material zurückgriff. Sein Werk darf nicht allein als Ausdruck einer ,antiquarischen Passion' verstanden werden, auch wenn diese - so Antonio Carile - „vergleichbar ist mit derjenigen, aus der die teilweise zeitgleichen KonstantinopelFührer hervorgegangen sind oder die verschiedenen Patria, die bereits zwischen dem 8. und 9. Jahrhundert nach dem Vorbild der Parastaseis syntomoi chronicai enstanden“. ${ }^{30}$ Das große annalistische und prosopographische Werk des Agnellus gehört zu den wichtigsten Zeugnissen, die es erlauben, den Geist zu verstehen, mit dem sich viele in den Kathedralschulen in einer Kultur der Rhetorik ausgebildete Kleriker den sichtbaren materiellen Quellen näherten, diese ausführlich benutzten und so überlieferten.

\section{Transformationen am Ende der Spätantike}

An diesem Punkt kann man beginnen, eine Reihe von Überlegungen anzustellen, indem man auf die epigraphische Praxis in der Zeit vor der Mitte des 6. Jahrhunderts blickt. Diese markiert letztlich in jeglicher Hinsicht für die Schriftkultur - nicht nur die inschriftliche - das langsame und allmähliche Abbrechen der römisch-antiken Tradition, wodurch meiner Meinung nach die epigraphische Produktion des gesamten Mittelalters geprägt ist.

Die Untersuchung befasst sich im Folgenden mit dem Rückgang der epigraphischen Texttypen seit dem Beginn des Mittelalters. Schon erwähnt wurde die Reduzierung auf funerale Zusammenhänge, obwohl zahllose römische Beispiele einge-

28 Bottazzi 2016, http://www.e-codices.unifr.ch.

29 Holder-Egger 1878, 265-391.

30 Carile 1992, 373-378. 
meißelter, gekratzter oder aufgemalter Schrift in unterschiedlichsten Verwendungszusammenhängen immer noch vor aller Augen standen.

In Rom sowie in allen Städten des Imperiums ,gab es wirklich viel zu lesen“. ${ }^{31}$ Die Kunstwerke, Gebäude und alle Bauten von öffentlicher Bedeutung haben in dieser Welt Inschriften aller Art zur Schau gestellt. Die Mauern der Straßen und Foren waren bedeckt mit eingravierten Graffiti oder aufgemalten Schriften (Dipinti) aus der Hand einer Bevölkerung, deren im Vergleich zu früheren Jahrhunderten höherer Alphabetisierungsgrad es ihnen erlaubte, ausführlich Liebschaften, sportliche Leistungen oder verschiedene Feindschaften im Medium der Inschrift zu äußern und zu feiern, lokale Politiker zu unterstützen, in den Inschriften ihren cursus zu dokumentieren oder sie als Wohltäter zu preisen. ${ }^{32}$ Inschriften erinnerten an Erdbeben und andere Naturkatastrophen, wie gleichzeitig auch an die durch sie notwendig gewordenen Restaurierungsarbeiten. Bewohner der Städte lasen in ihnen den Namen des eigenen patronus, der sie in Rom unterstützen würde. ${ }^{33}$ Durch die epigraphischen Texte wurden ferner Mietwohnungen angeboten, Belohnungen für den Fund verlorener oder gestohlener Gegenstände oder für die Ergreifung geflohener Sklaven versprochen. Aufgemalte Schriften kündigten Theater- und Zirkusvorstellungen an und luden in tabernae ein. Lange Auflistungen warben für den Verkauf von Waren und nannten deren Preis. Auf den Friedhöfen vor der Stadt wurden Grabinschriften ausgestellt, die oft in aediculae eingraviert und mit plastischen Portrait-Büsten geschmückt waren. Sie zogen die Aufmerksamkeit der Vorbeikommenden an und veranlassten diese dazu, anzuhalten, um zu lesen, den Verstorbenen die Ehre zu erweisen und sich auszuruhen. Einzelne Inschriften, die auf den Grabmonumenten ausgestellt waren, konnten außerdem über die Form des errichteten Mausoleums und die rechtlichen Umstände des Erwerbs der für das Grab bestimmten Fläche Auskunft geben. Oftmals wurden Ausschnitte aus dem Testament des Verstorbenen eingemeißelt, welche die Errichtung des Grabmonuments oder einer Stiftung betrafen. Letztere entstand durch die Hinterlassenschaft von Geldbeträgen, Ländereien und aedificia zur Kostenunterstützung für die Grabpflege und die Totenfeiern. Auch enthielten die Inschriften die Namen der Enterbten und die Auflistung der Flüche (maleficia) gegen mögliche Grabschänder oder diejenigen, die den Friedhofsbereich mit einer Latrine verwechselt hätten. ${ }^{34}$ An den Grenzbereichen der Nekropolen verwiesen schließlich Platten auf den Umfang der Friedhofsfläche und ermahnten in allgemeiner Weise die Vorbeigehenden, den den Verstorbenen gewidmeten Bereich zu ehren.

Jedoch waren nicht nur die Großstädte in Republik und Kaiserzeit von öffentlich ausgestellter Schrift geprägt. Auch das zugehörige Territorium sowie die kleineren

31 Buonopane 2009, 15.

32 Breit erforscht in Actes du Xe Congrès international d'épigraphie grecque et latine 1992; für die augusteische Zeit siehe Alföldy 1997, 293-331.

33 Galli/Molin/Scaroina 2007/08, 9-32.

34 Buonopane 2009. 
Zentren waren Orte epigraphischer Inszenierungen, und sie blieben es zumindest bis zum Ende des 6. Jahrhunderts. Die Straßen waren von Meilensteinen gesäumt, die Namen von Kaisern, Magistraten und großzügigen Geldgebern trugen. Auf den Landgütern markierten Inschriften-Stelen die Besitzgrenzen, während Votivsteine auf kleine rurale Heiligtümer hinwiesen, die den Göttern geweiht waren. Auf dem Höhepunkt von Kriegs- und Krisenzeiten schützte die Gesetzgebung Inschriften gegenüber der systematischen Wiederverwendung und Spoliierung. ${ }^{35}$

Die Anzahl erhaltener republikanischer und kaiserzeitlicher Inschriften wird auf etwa 300.000-400.000 Exemplare geschätzt - eine durchaus beachtliche Zahl, die aber immer noch annähernd nur zwei oder drei Prozent der gesamten epigraphischen Produktion entspricht, wie sich auf der Basis archäologischer Befunde und der Heranziehung sekundärer Überlieferung in hypothetischer Form schätzen lässt. Der sekundären Überlieferung, die hauptsächlich aus mittelalterlichen und modernen Corpora sowie den zuvor erwähnten epigraphischen Sammlungen besteht, verdanken wir trotz ihrer Lückenhaftigkeit einerseits die Möglichkeit, die Bedeutung von Inschriften sowohl für die römische Antike als auch für das Frühmittelalter zu bestimmen. Andererseits belegen diese Sammlungen die angesprochene epigraphische Kontinuität, die es trotz des erwähnten quantitativen und qualitativen Rückgangs zwischen Spätantike und Mittelalter gab. Tatsächlich zeigen die numerischen Schätzungen, dass ab der zweiten Hälfte des 4. Jahrhunderts in vielen Zentren außerhalb Roms die epigraphische Praxis immer mehr abnahm, auch wenn ein regelrechter gap - soweit heute bekannt - erst zwischen dem Ende des 6. und dem Ende des 8. Jahrhunderts einsetzte $^{36}$ - und dies, obwohl die Epigraphik zwischen dem 3. und 4. Jahrhundert, insbesondere nach der ,konstantinischen Wende' auch für die Christen weiterhin das geeignetste Medium war, um individuelle sowie kollektive Memoria im funeralen Kontext zu sichern. ${ }^{37}$

Schon seit der Konstantinischen Zeit war ein Rückgang der auf hohe Verwaltungspositionen bezogenen epigraphischen Produktion mit der Folge einer geringen Sichtbarkeit dieser Personen zu beobachten. Dieser Rückgang verstärkte sich dann noch zur Zeit der „Regionalisierung“ der Prätorianerpräfekturen sowie des komplexeren und „drastischeren Regimewechsels von 337“.38 Letzterer lässt sich zudem auf weitere Spannungen und Krisen zurückführen, unter denen nur die Konflikte zu nennen sind, die auf der Ebene der Religion Imperium und christliche Kirche in

35 De Rubeis 2010, 709-710.

36 Die Schätzungen stammen aus Carletti 2000, 81-88, ders. 2008 und De Rubeis 2007, 705-727. Der Zeitraum, in dem der epigraphische gap festgestellt wird, entspricht in Italien der Zeit von den gotischen Kriegen über die Langobardenzeit bis zu den Eroberungen der Karolinger.

37 Cracco Ruggini 1996, 170-175. Für einen Überblick über die Forschung zur christlichen Epigraphik: De Rubeis 2007; Carletti 2008.

38 Porena 2006, 355. 
Opposition zueinander brachten, während letztere in ihrem Inneren unter diversen theologischen Kontroversen litt.

Bei aller Vorsicht, die man stets bei der Interpretation von abbrechender oder zurückgehender Überlieferung walten lassen muss, die ich schon an anderer Stelle und für andere Inschriftenbestände bemerkt habe, ${ }^{39}$ ist es doch häufig möglich, den abnehmenden Gebrauch von Inschriften mit der objektiven Schwierigkeit der Menschen zu erklären, Nachrichten von gehobenem und feierlichem Inhalt in Momenten von Spannung und Gefährdung zu verfassen.

Unter diesen diversen ,schwierigen' Momenten zwischen dem 3. und 4. Jahrhundert wird man sicherlich die unterschiedlichen Phänomene einreihen können, die $\mathrm{zu}$ Veränderungen in den civitates führten, und hier vor allem der von Peter Brown beschriebene Aufstieg der Kirche ,auf einer langsam steigenden Flut wohlhabenden Patronats“. ${ }^{40}$ Die Arbeiten von Charles Pietri über den christlichen Euergetismus sowie die von Yvette Duval und Luce Pietri über das Verhältnis zwischen dem Euergetismus des 4. und 5. Jahrhunderts und der Epigraphik sind hier sehr instruktiv. ${ }^{41}$ So wird der Beginn der großen Epoche des christlichen Euergetismus in das 4. Jahrhundert datiert. Gleichzeitig beziehen sich reiche Bürger aus dem Westen, etwa aus dem römischen Afrika zur Zeit des Augustinus (354-430), in den von ihnen gesetzten Inschriften auf die ungleich banalere amor civium, wenn sie Geld zwar immer noch in der Rolle des Euergeten stiften, jedoch nicht mehr für öffentliche Bauvorhaben, sondern zur Abhaltung von prunkvollen Schauspielvorführungen, Zirkusrennen oder Kämpfen. ${ }^{42}$ Die normativen Verweise im Codex Theodosianus, die in der Historiographie oftmals erwähnt werden, müssen im Kontext einer Reduktion des Kapitals betrachtet werden, über das maßgebende Persönlichkeiten verfügten, die institutionell im römisch-staatlichen ,Apparat' eingegliedert und weiterhin an die Tradition des Euergestimus gebunden waren. Der Codex richtet sich gegen die persönliche Verwendung epigraphischer Schriften durch opportunistische Statthalter. Wenn diese als Gegenleistung zu einem Bau, der durch öffentliche Gelder gedeckt war, in der Inschrift ihren Namen statt den des Kaisers hätten eingravieren lassen, so hätten sie nach dem Gesetz wegen Majestätsbeleidigung bestraft werden müssen. ${ }^{43}$ Währenddessen drängten die Geistlichen dahin, dass die Privatgelder reicher Christen nicht mehr in das öffentliche Bauwesen, sondern zur Steigerung des Wohlstandes des hohen Klerus oder zum Bau von Märtyrerkirchen und Klöstern eingesetzt würden. Dies bezeugt eine am 30. Juli 370 von den Kaisern Valentinian, Valens und Gratian veröffentlichte Verfügung, die an den zu dieser Zeit in Rom noch angefochtenen Papst Damasus gerichtet war. In dieser, die in den Kirchen angeschlagen und verlesen

39 Bottazzi 2012, 132.

40 Brown 1983, 50.

41 Pietri 1976, Bd. 1, 581; Duval/Pietri 1997, 371-396.

42 Arcuri 2008, Bd. 2, 1049-1061.

43 Cod. Theo. XV, I,31, 394; De Rubeis 2006, $101 f$. 
werden sollte und die schließlich in den Codex Theodosianus (16.2.20) aufgenommen wurde, wird berichtet, dass „Witwen und Jungfrauen umgarnt wurden, um von diesen Schenkungen und testamentarische Legate zu erhalten“. ${ }^{44}$

\section{Der Einfluss von Papst Damasus}

Mit Blick auf die Untersuchung der epigraphischen Kontinuität zwischen Spätantike und Mittelalter sollte die Abkehr der gesellschaftlichen Mittelklasse von der epigraphischen Tradition weniger auf einen allgemeinen Kulturverfall zurückgeführt werden. Vielmehr ist auch - wie De Rubeis zurecht vorgeschlagen hat - der seit Beginn der Christianisierung durch die christliche Ideologie bestimmte grundlegende Wandel zu berücksichtigen, der dazu geführt hat, dass die christliche Bevölkerung ihrem Gott nicht mehr als einzelne Individuen, sondern als eine Gemeinde gegenübertrat - ein Faktor, der die Produktion von Funeral-Inschriften zweifellos signifikant bremste. Erst in einer zweiten Phase sollte die Sicherung christlicher Memoria wieder ein starkes individuelles Bedürfnis werden. ${ }^{45}$

Zudem darf De Rubeis zufolge nicht vergessen werden, dass die christliche Epigraphik eine sozial ausdifferenzierte Praxis darstellte, so dass der Begräbnisplatz für die vielen Armen auf lange Zeit die epigraphisch wenig ergiebige Gemeinschaftsgrablege war, während die Eliten, namentlich die Bischöfe, Gräber an Orten bevorzugten, die durch die Präsenz von Heiligen und Märtyrern ausgezeichnet waren. ${ }^{46}$ Diese Eliten bedienten sich zudem regelmäßig der eingravierten und häufig sichtbar ausgestellten Inschrift, ${ }^{47}$ wobei sich die damasianische Kapitalis als kanonisierte Schriftform durchsetzte: die im 4. Jahrhundert durch den Kalligraphen Filocalcus für Papst Damasus entwickelte elaborierte epigraphische Schrift, die dessen gesamte Reliquien-Erhebungs-Kampagne charakterisierte. ${ }^{48}$ In diesem Zusammenhang ist noch einmal der Einfluss zu betonen, den Papst Damasus, an den die erwähnte Verordnung von 370 gerichtet war, und den die Paläographen als einen Erneuerer der Schrift betrachten, auf den Wandel der Inschriften-Praxis im 4. Jahrhundert hatte.

Die Ergebnisse seines etwas mehr als 18-jährigen Pontifikats werden äußerst kontrovers bewertet. Glaubwürdige Quellen wie Ammianus Marcellinus, der in seinen Beurteilungen immer als sehr zuverlässig und bedacht betrachtet wurde, helfen, die historische Situation zu verstehen, in die Damasus mit seinem Streben nach der römi-

44 Barzanò 1993 (2), 359-366; [http://hdl.handle.net/10807/38609].

45 Carletti 2008.

46 De Rubeis 2006.

47 Picard 1988.

48 De Rubeis 2010, 717. 
schen Kathedra einzuordnen ist. ${ }^{49}$ Damasus' Pontifikat war das Ergebnis von Konflikten zwischen bewaffneten Gruppen, die nach der Macht strebten. In der Tat ist sein Pontifikat durch das Schisma charakterisiert, das seine umstrittene und problematische Weihe auslöste. Während Ammianus Marcellinus den Gegenspieler Ursinus zu unterstützen scheint, wurde Damasus hingegen von zu ihrer Zeit so herausragenden kirchlichen Autoren wie Hieronymus, Ambrosius, Rufinus, Socrates und Sozomenos für den legitimen Papst gehalten. Jedenfalls verkörperte Damasus angemessen die geistlichen Eliten der 60er-Jahre des 4. Jahrhunderts. Diese wurden von verschlagenen römischen Diakonen gebildet, die angesichts eines Aufstiegs auf den Bischofsstuhl von Ostia und schließlich auf die römische Kathedra danach eiferten, ihre Nähe zum Papst als Sprungbrett zu nutzen, um politische Unterstützung im Senat, reichliche Finanzierungen und schließlich gar die kaiserliche Unterstützung zu erhalten. Damasus war tatsächlich ein unsicherer, wenn nicht gar opportunistischer Angehöriger des römischen Diakonenkollegiums. In den Jahren, die er im Gefolge seines Vorgängers Liberius sowie an der Seite des Gegenpapstes Felix verbrachte, fand er die richtigen Unterstützer und die geeigneten finanziellen Mittel aus der römischen Senatorenaristokratie sowie schließlich die sichere Unterstützung des Kaisers. Diesem verdankte er, dass seine Stellung in Rom und im Lateran erstaunlicherweise gesichert blieb, obwohl gegen ihn Anklagen wegen gewaltsamer Übergriffe vorlagen. Indem Ammianus Marcellinus ihm das Bild des Provinzbischofs gegenüberstellt, der sich mit einem sehr kargen Mahl zufriedengibt, bescheiden gekleidet ist und seine Augen immer zu Boden gesenkt hat, schildert er Damasus als einen Selbstdarsteller, der Bequemlichkeiten, Prunk und Festmähler einer gehobenen Gesellschaft liebt, zu der ebenjene Matronen gehören, die den Bischof mit ihren Spenden bereichern. Im umfangreichen Beitrag von Carlo Carletti über Damasus steht indes das Fehlen von theologischer Substanz den grundlegenden Errungenschaften für das Papsttum gegenüber, die Damasus in großen Teilen seiner Amtszeit beschäftigt haben müssen. ${ }^{50}$ Diese war von Beginn an durch eine literarische Produktion gekennzeichnet, der wir noch begegnen werden, sowie durch das Interesse an jährlich stattfindenden Synoden und Konzilien, die für die Diskussionen innerhalb der christlichen Kirche von Bedeutung waren. Für die christliche Kirche des 4. Jahrhunderts war Damasus eine Schlüsselfigur, wenn er auch nicht auf die gleiche theologische Ebene wie Ambrosius und Hieronymus gestellt werden kann. Hieronymus wirkte in den Jahren 382 bis 385 als Berater und Sekretär für Damasus. Im Hintergrund verfolgte Hieronymus die gleichen kirchenpolitischen und pastoralen Linien wie der Papst. Einige zentrale kirchengeschichtliche Entwicklungen seien angedeutet: die sich im 4. Jahrhundert verbessernde Finanzlage aufgrund der euergetischen Freigebigkeit reicher Christen, die Neudefinition des Kultes von Märtyrern und Heiligen, die christliche Adaption

49 Amm. XXVII, 3, 12-13; 14-15. Vgl. Carletti 2000.

50 Carletti 2000. 
paganer Kulte und Heiligtümer sowie die neue Bautätigkeit im Bereich von Kirchen und Begräbnisstätten. All dies wurde von epigraphischer Kommunikation begleitet, da die Inschrift zwar einerseits das geeignete Mittel zur Vermittlung weltlicher Gesetzgebung war, andererseits aber, wie bereits in vorchristlicher Zeit, auch zum Zeugnis der spannungsreichen Einstellung der Christen gegenüber Tod und Jenseits wurde. ${ }^{51}$ Carlo Carletti schreibt in seiner Damasusbiographie dem Papst und seinem Klerus das Verdienst zu, die epigraphische Monumentalschrift verwendet $\mathrm{zu}$ haben, um im Lateransbaptisterium, an San Lorenzo in Damaso, San Ippolito und schließlich in der Felix gewidmeten Inschrift das Konzept der „sedes apostolica“ öffentlich zu verbreiten und zu etablieren. ${ }^{52}$ Ich möchte daher die Bedeutung unterstreichen, die Damasus für die radikale Wende der christlichen Epigraphik hatte, indem er seine inschriftlich umgesetzte literarische Produktion in die Kampagne zur Wiederentdeckung der Märtyrerreliquien sowie die Restaurierung der Begräbnisstätten integrierte..$^{53}$ Die zur Erinnerung an die Märtyrer angefertigten und an den Kirchen und Begräbnisorten angebrachten Grabinschriften folgten dabei dem von Filocalus entwickelten neuen graphischen System. Die neue Schrift, die wegen ihrer gerundeten Serifen gut erkennbar ist, unterschied sich deutlich von der epigraphischen Majuskel, obwohl sie aus dieser hervorgegangen war. Sie wird zur Schrift der Verherrlichung der Märtyrer, wohingegen die epigraphische Majuskel die Schrift für die Grabinschriften der Päpste blieb.

Damasus betreffend wird - wie bereits erwähnt - gewöhnlich unter einem paläographischen Gesichtspunkt nur die symbolische und graphische Hervorbringung dieser neuen Schrift betont, wohingegen meines Erachtens die am Ende des 4. Jahrhunderts einsetzenden Folgen des starken pastoralen Impulses hin zur kultischen Verehrung der Märtyrer übersehen werden. Diese haben die Bestattungspraxis der kirchlichen Eliten tiefgreifend beeinflusst, da diese nun immer stärker nach einer Bestattung möglichst nah an den Körpern der Heiligen strebten, nachdem die Kirchen und vor allem deren Innenräume zu Begräbnisorten geworden waren. ${ }^{54}$ So entstand jene Differenz der Schriftkulturen, die zu einer sichtbaren Unterscheidung der Menschen hinsichtlich ihrer sozialen Herkunft führte. ${ }^{55}$ Tatsächlich wurde der Gebrauch von Inschriften den Laien verschlossen. Der Abstand zwischen diesen und der neuen christlichen Lehre wuchs, die sich auf die wenigen Märtyrer stützte, auf die Auserwählten (Gregor von Tours) oder die ,Prädestinierten' (Augustinus), in etwa den Bischöfen entsprechend, wodurch viele Menschen ausgeschlossen wurden, die sich durch gute Taten ausgezeichnet hatten. ${ }^{56}$ Oft werden zudem die Wirkungen nicht ausreichend

51 Brown 1981, 101.

52 Carletti 2000.

53 Carletti 2000.

54 Picard 1988, 1; Tabacco 2000, 3-12; Bottazzi, 2012, 77.

55 De Rubeis, 2006.

56 Brown 1981, 104-105. 
berücksichtigt, die die zahlreichen Epigramme von Damasus selbst, aber auch von Ambrosius, Hieronymus, Paulinus von Nola, Augustinus, Sidonius Apollinaris und Venantius Fortunatus auf die hochmittelalterliche epigraphische Praxis hatten. Papst Damasus bzw. dem Bischof von Ostia verdanken wir zahlreiche Epigramme und Märtyrer-Elogen, zudem Inschriften, die zur Erinnerung an seine Bautätigkeiten angefertigt wurden, Elogen auf wichtige biblische Gestalten, aber auch eine interessante Gruppe von Inschriften, die an die ,teuren' Vorfahren erinnerten und die eine nennenswerte Zahl von Nachahmern fanden. Die Epitaphe für den Vater Antonius, die Mutter Lorenza, die Schwester Irene, für Freunde und ,seine‘ Märtyrer wurden schon bald und für einen langen Zeitraum nachgeahmt. ${ }^{57}$ Die ,post-Damasus'-Zeit wird nach dem derzeitigen Kenntnisstand durch etwa sechzig Inschriften repräsentiert, worunter sowohl Originale zu zählen sind, die an unterschiedlichen Standorten aufbewahrt werden, als auch Abschriften aus dem 7. Jahrhundert sowie aus mittelalterlichen Sammlungen, die zwischen dem 8. und 12. Jahrhundert verfasst wurden. Genannt seien die Lorscher Sammlung (9.-10. Jahrhundert) sowie die Sammlungen aus Tour (12. Jahrhundert) und Verdun (7.-9. Jahrhundert).

Zeugnisse für die Nachahmung damasianischer Epigraphik findet man etwa in Mailand, wo die epigraphische Kontinuität zwischen Spätantike und Mittelalter deutlicher zu erkennen ist als in anderen Städten. Dort ließ der Subdiakon und spätere Erzbischof Walpertus Mitte des 10. Jahrhunderts eine antike Urne aus römischer Zeit bearbeiten, um die Überreste der „liebenswerten“ Eltern zu sammeln. Auf der Urne ließ er in elegantem Latein verfasste Texte eingravieren, die an sie erinnerten und von seiner Liebe zu ihnen berichteten, ohne zugleich einen Hinweis darauf auszulassen, dass er mit dieser Handlung seinen Vorgänger nachahmte. Ein Jahrhundert zuvor hatte der levita Andreas, „captus amore“, seine eigenhändigen epigraphischen Bemühungen seinem geliebten Erzbischof Anspertus angeboten. Währenddessen standen Märtyrer und Heilige sowie ihre Reliquien von der Spätantike bis zum Mittelalter weiterhin im Fokus der kirchlichen Aufmerksamkeit, da sie sich schließlich nicht zuletzt zu einem großen Geschäft entwickelt hatten. Während Märtyrer im Osten ab dem 4. Jahrhundert erhoben und erneut beigesetzt wurden, erfolgte dies in Rom sowie im gesamten Westen erst seit dem 6. Jahrhundert. In Nachahmung entsprechender Praktiken aus Afrika wird das Hochmittelalter lange Listen unbekannter Märtyrer anlegen, die in den verschiedenen römischen Katakomben beigesetzt und später erhoben wurden, um - etwa in S. Prassede - verehrt zu werden. Später wird dort eine Weihe-Inschrift angefertigt, die sicherlich nicht der damasianischen ähnelt, die jedoch auf Ewigkeit und öffentlich sichtbar an die 2300 Märtyrer und an Paschalis I. erinnert, der zwischen 817 und 824 diese Sammlung angeordnet hatte. ${ }^{58}$

57 Testini 1980, 2. Aufl., 464-465; Carletti 2000, 351.

58 Bottazzi 2012, 30, 57, 99, 108, 111, 112. 


\section{Literaturverzeichnis}

Alföldy, Géza (1997), „Euergetismus und Epigraphik in der Augusteischen Zeit“, in: Michel Christol u. Olivier Masson (Hgg.), Actes du Xe congrès international d'épigraphie greque et latine, Nîmes, 4-9 octobre 1992, Paris (Publications de la Sorbonne; Séries Histoire Ancienne et médiévale, 42), 293-331.

Porta, Giuseppe (Hg.) (1979), Anonimo romano: Cronica (Classici 40), Mailand.

Arcuri, Rosalba (2008), „Il “buono“ e il „cattivo“ uso delle ricchezze nell'Africa romana tardo antica“, in: Juliàn Gonzàles, Paola Ruggeri, Cinzia Vismara u. Raimondo Zucca (Hgg.), Africa Romana. Le ricchezze dell'Africa. Risorse, produzioni, scambi, Rom, Bd. 2, 1049-1061.

Auerbach, Erich (2007 ital. Ausg.), Lingua letteraria e pubblico nella tarda antichità latina e nel Medioevo, Mailand.

Auerbach, Erich (1958), Literatursprache und Publikum in der lateinischen Spätantike und im Mittelalter, Bern.

Barzanò, Alberto (1993), „La questione dell'arricchimento dei vescovi e del clero da Cipriano a Damaso: tra polemica anticristiana, autocritica ecclesiale e legislazione imperiale“, in: Rivista di Storia della Chiesa in Italia 47 (2), 359-366; http://hdl.handle.net/10807/38609 (Stand 10.11.2018).

Bottazzi, Marialuisa (2006), „Tra Papato e Impero: l'uso dell'epigrafia nei secoli XI e XII a Viterbo“, in: Studi medievali 3, 305-360.

Bottazzi, Marialuisa (2007), „Frater Jacobus, Jacobus abbas. Impero, cistercensi e celebrazione monumentale nel conflitto milanese, 1160-1183“, in: Studi medievali 3, 271-306.

Bottazzi, Marialuisa (2012a), Italia medievale epigrafica. L'alto medioevo attraverso le scritture incise (secc. IX-XI) (Studi 08), Triest.

Bottazzi, Marialuisa (2012b), „Città e scrittura epigrafica“, in: Miriam Davide (Hg.), Identità cittadine e aggregazioni politiche in Italia, secoli XIXV, Convegno di studio (Trieste, 28-30 giugno 2010) (Atti 03), Triest, 275-302.

Bottazzi, Marialuisa (2012c), „Ancora sulle epigrafi di Collescipoli del 1094. Per una storia delle chartae lapidariae“, in: Bollettino della Deputazione di storia patria per l'Umbria 109, 501-522; u. „Collescipoli, Chiesa di San Stefano, 1094, Trascrizione epigrafica dell'iscrizione“, in: ebd. (12 fuori testo), 501-522.

Bottazzi, Marialuisa (2015), „Nell'anno del Signore 1213, il popolo di Siena“, in: Duccio Balestracci (Hg.), Monteriggioniottocento 1214-2014 (Atti del Convegno, Abbadia a Isola, 17 ottobre 2014), Siena, 47-60.

Bottazzi, Marialuisa (2016), „L'epigrafia dell'Italia Comunale: evidenze positive e negative“, in: Arturo Calzona u. Glauco Maria Cantarella (Hgg.), Dalla Res Publica al Comune. Uomini, istituzioni, pietre dal XII al XIII secolo (Atti del convegno internazionale di studi, Mantova 3-5 dicembre 2014), Mantua, 25-53.

Bottazzi, Marialuisa, „Gli statuti epigrafici dell'Italia centro-settentrionale (secc. XII-XIV)“, in: Didier Lett (Hg.), (im Druck bei Publications de la Sorbonne).

Brown, Peter (1983), Il culto dei santi. L'origine e la diffusione di una nuova religiosità, Turin.

Buonopane, Alfredo (2009), Manuale di epigrafia latina (Beni culturali 33), Rom.

Cammarosano, Paolo (1999), Nobili e re. L'Italia politica dell'alto medioevo, Bari.

Cammarosano, Paolo (2000, 6. Aufl.) Italia medievale. Struttura e geografia delle fonti scritte, Rom. Capasso, Bartolommeo (1892), Monumenta ad Neapolitani Ducatus Historiam Pertinentia, Neapel.

Carile, Antonio (1992), „Agnello storico“, in: Storia di Ravenna 2, 373-378.

Carletti, Carlo (2008), Epigrafia dei cristiani in occidente dal III al VII secolo. Ideologia e prassi (Inscriptiones Christianae Italiae, Subsidia 6), Bari.

Carosi, Attilio (1986), Le epigrafi medievali di Viterbo, Viterbo. 
Cavallo, Guglielmo (1988) „Le tipologie della cultura nel riflesso delle testimonianze scritte“, in: Bisanzio, Roma e l'Italia nell'Alto Medioevo, Spoleto, 467-516.

Cavallo, Guglielmo/Mango, Cyril A. (Hgg.) (1995), Epigrafia medievale greca e latina, ideologia e funzione. Atti del Seminario di Erice (12-18 settembre 1991), Spoleto.

Cracco Ruggini, Lellia (1996), Storia Antica. Come leggere le fonti, Bologna.

De Rubeis, Flavia (2000), „Tradizione epigrafica in Paolo Diacono“, in: Paolo Chiesa (Hg.), Paolo diacono. Uno scrittore fra tradizione longobarda e rinnovamento carolingio, Atti del Convegno Internazionale di Studi, Cividale del Friuli - Udine, 6-9 maggio 1999, Udine, 139-173.

De Rubeis, Flavia (2003), „La tradizione epigrafica longobarda nei ducati di Spoleto e Benevento“, in: I Longobardi dei ducati di Spoleto e Benevento, Atti del XVI Congresso Internazionale di Studio sull'Alto Medioevo (Spoleto, 20-23 ottobre 2002 - Benevento, 24-27 ottobre 2002), Spoleto, 481-506.

De Rubeis, Flavia, (2006), „La scrittura delle élites tra crisi e rinnovamento“, in: François Bougard, Laurent Feller u. Regine Le Jan (Hgg.), Les élites au haut moyen age crises et renouvellements, Tourhout, 99-126.

De Rubeis, Flavia (2010), „La capitale damasiana a Tours: esperimenti ed effimere primavere“, in: Scripta. An International journal of codicology and paleography 3, Pisa - Rom, 57-71.

Duval, Yvette-Pietri, Luce (1997), „Évergétisme et épigraphie dans l'occident chrétien (IV-VI s.)“, in: Michel Christol u. Olivier Masson (Hgg.), Actes du Xe congrès international d'épigraphie greque et latine, Nîmes, 4-9 octobre 1992 (Publications de la Sorbonne; Séries Histoire Ancienne et médiévale 42), Paris, 371-396.

Feissel, Denis (1995), „Épigraphie et constitutions impériales: aspects de la publications du droit à Byzance“, in: Guglielmo Cavallo u. Cyril Mango (Hgg.), Epigrafia medievale greca e latina, ideologia e funzione. Atti del Seminario di Erice (12-18 settembre 1991), Spoleto, 67-98.

Felle, Antonio Enrico (2015), „Esporre la Scrittura. L'uso dei testi biblici nelle epigrafi d'ambito pubblico tra Tarda Antichità e prima età bizantina (secoli IV-VIII)“, in: Antiquité Tardive. Revue Internationale d'Histoire et d'Archéologie (IVe-VIle siècle) 23, 353-370.

Ferraiulo, Daniele (2013), Tra canone e innovazione. Lavorazione delle epigrafi nella „Langobardia minor" (secoli VIII-X), Florenz.

Flussin, Bernard (2007), „IV. Le strutture della Chiesa imperiale“, in: Cécile Morrisson (Hg.), Il mondo bizantino. L'impero romano d'Oriente (341-641), ital. Ausg. hg. von Silvia Ronchey u. Tommaso Braccini, Turin, 119-151.

Franceschini, Adriano (1969), I frammenti epigrafici degli statuti di Ferrara del 1173 venuti in luce nella cattedrale, Ferrara

Galli, Paolo / Molin, Diego/Scaroina, Luigi (2007/08), „Tra fonti storiche e indizi archeologici. Terremoti a Roma oltre la soglia del danno“, in: Rivista dell'Istituto Nazionale d'archeologia e storia dell'arte 62-63, 9-32.

Holder-Egger, Oswald (Hg.) (1878), Agnelli qui et Andreas Liber pontificalis ecclesiae Ravennatis, in: MGH SS.rer.lang., 265-391.

Lambert, Chiara (2008), Studi di epigrafia tardo antica e medievale in Campania. Bd. I. Secoli IV-VII, Florenz.

Lambert, Chiara (2012), „Documento-monumento: della duplice natura delle fonti epigrafiche in esempi della "Langobardia minor““', in: Fiorillo Rosa u. Chiara Lambert (Hgg.), Medioevo letto, scavato, rivalutato. Studi in onore di Paolo Perduto, Florenz, 99-114.

Lepellet, Claude (1997), „Évergétisme et épigraphie dans l'antiquité tardive: les province de langue latine“, in: Michel Christol u. Olivier Masson (Hgg.), Actes du Xe congrès international d'épigraphie greque et latine, Nîmes, 4-9 octobre 1992 (Publications de la Sorbonne; Séries Histoire Ancienne et médiévale 42), Paris, 335-352.

Lepore, Paolo (22012), „Rei Publicae Polliceri“. Un'indagine giuridico-epigrafica, Mailand. 
Morrisson, Cécile (2007), „La continuità dell'Impero romano in Oriente; Gli avvenimenti: prospettiva cronologica“, in: Cécile Morrisson (Hg.), Il mondo bizantino. L'impero romano d'Oriente (341-641), ital. Ausg. hg. von Silvia Ronchey u. Tommaso Braccini, Turin, 5-52.

Orlandi, Silvia (2006), „Le iscrizioni del Colosseo come base documentaria per lo studio del senato tardo antico“, in: Rita Lizzi Testa (Hg.), Le trasformazioni delle élites in età tardo antica, Atti del Convegno Internazionale (Perugia, 15-16 marzo 2004), Rom, 311-324.

Petrucci, Armando (1995), Scritture ultime, ideologia della morte e strategie dello scrivere nella tradizione occidentale, Turin.

Petrucci, Armando (1985), „Potere, spazi urbani, scritture esposte: proposte ed esempi“, in: Culture, et ideologie dans la genèse de l'État moderne (14-17 ottobre, 1984), Rom, 85-97.

Picard, Jean-Charles (1988), Le souvenir des évêques. Sépultures, listes épiscopales et culte des évêques en Italie du Nord des origines au Xe siècle, Rom.

Pietri, Charles (1976), Roma cristiana, 2 Bde. (Bibliothèque de l'Ecole française d'Athènes et Rome 224), Paris.

Porena, Pierfrancesco (2006), „Trasformazioni istituzionali e assetti sociali: i prefetti del pretorio tra III e IV secolo“, in: Rita Lizzi Testa (Hg.), Le trasformazioni delle élites in età tardo antica, Atti del Convegno Internazionale (Perugia, 15-16 marzo 2004), Rom, 325-356.

Rotili, Marcello (2015), „Testimonianze monumentali e archeologiche“, in: Gabriele Archetti (Hg.), Desiderio. Il progetto politico dell'ultimo re longobardo. Atti del Primo convegno internazionale di studio (Brescia, 21-24 marzo 2013), Spoleto, Fondazione Centro italiano di studi sull'Alto Medioevo, (Centro studi longobardi. Convegni 1), 75-144.

Rouché, Charlotte (1997), „Benefactors in the Late Roman period: the Eastern Empire“, in: Michel Christol u. Olivier Masson (Hgg.), Actes du Xe congrès international d'épigraphie greque et latine, Nîmes, 4-9 octobre 1992 (Publications de la Sorbonne; Séries Histoire Ancienne et médiévale 42), Paris, 353-368.

Sanazzaro, Marco (2006), „Epigrafia e città“, in: Andrea Augenti (Hg.), Le città italiane tra la tarda Antichità e l'alto Medioevo, Atti del convegno (Ravenna, 26-28 febbraio 2004), Florenz, 81-96.

Santiago Fernàndez, Javier (2009), „El hábito epigráfico en la Hispania visigoda“, in: Galende Juan Carlos Díaz, u. Javier de Santiago Fernández (Hgg.), VIII Jornadas Científicas sobre Documentacion de la Hispania altomedieval (siglos VI-X), Universidad Complutense de Madrid-Dpto. de Ciencias y Técnicas Historiográficas, Madrid, 291-344.

Tabacco, Giovanni (2000), Le ideologie politiche del medioevo, Turin.

Testini, Pasquale (21980), Archeologia cristiana. Nozioni generali dalle origini alla fine del secolo VI, propedeutica-topografia cimiteriale - epigrafia - edifici di culto (con aggiunta di indice anlit. $e$ append. bibliografica), Bari.

Wörrle, Michael (1988), Stadt und Fest im kaiserzeitlichen Kleinasien: Studien zu einer agonistischen Stiftung aus Oinoanda (Vestiga 39), München. 